\title{
FAT OVERLOAD FOCAL SEIZURES
}

Two 9-year-old patients receiving fat emulsion therapy (FET) who presented with focal seizures and other neurologic complications are reported from the Baylor College of Medicine, Neurology Service, Texas Children's Hospital, Houston, TX. FET (Intralipid) was administered during treatment of aplastic anemia by bone marrow transplantation in one and because of poor oral intake in another with cystic fibrosis. Focal seizures were associated with a hemiparesis, weakness, and altered mental status. CT of one child showed bilateral hypodensities, more prominent in one hemisphere. Both patients died of pneumonia. Autopsy findings included cerebral endothelial and intravascular lipid deposition, and multiple areas of necrosis and hemorrhage. (Schulz PE et al. Neurological complications from fat emulsion therapy. Ann Neurol May 1994;35:628-630). (Respond: Dr Schulz, Department of Neurology, NB-302, Baylor College of Medicine, One Baylor Plaza, Houston, TX 77030).

COMMENT. A rapid rise in triglyceride levels may have contributed to the lipid deposition in brain endothelium and onset of seizures. Early recognition of the fat overload syndrome may allow prompt withdrawal of fat emulsion therapy and reversal of neurologic symptoms.

\section{HEADACHE}

\section{INTERNATIONAL HEADACHE SOCIETY CRITERIA}

A prospective study involving 72 children with recurrent headache, designed to determine whether the diagnosis of headache type made intuitively by each of 4 neurologists would have met the IHS diagnostic criteria, is reported from the Department of Pediatrics and Child Health, Children's Hospital and University of Manitoba, Winnipeg, and the Division of Pediatric Neurology, Children's Hospital, Calgary, Canada. The intuitive clinical diagnoses were as follows: migraine without aura (44 cases), migraine with aura (11), migraine and tension-type (11), tension-type (3), post-traumatic (2), and sinus (1). Features considered were location (unilateral or bilateral), quality (pulsating or pressing), intensity, exercise aggravation, nausea, vomiting, photophobia, phonophobia, and age. Dietary triggers were recognized in 8 of 44 children with migraine without aura, in 2 of 11 with migraine with aura, in 3 of 11 with combined migraine and tension headache, and in none with tension headaches. Family history of migraine in a firstdegree relative occurred in 26 of 44 with migraine without aura, and 5 of 11 with migraine with aura. The intuitive diagnosis was completely concordant with the IHS criterion diagnosis in $61 \%$, partially concordant in $31 \%$ and at complete variance in $8 \%$. (Seshia SS et al. International headache society criteria and childhood headache. Dev Med Child Neurol May 1994;36:419-428). (Respond: Professor Shashi S Seshia, AE 208, Children's Hospital, 840 Sherbrook St, Winnipeg, Manitoba R3A 1S1, Canada).

COMMENT. The authors concluded that the IHS criteria (1988), intended mainly for adults, can also be applied to children with recurrent headaches, but with some reservations. Revisions to the criteria should take into consideration the inability of children to describe the qualities and location of pain precisely. Children's reports of the quality of headache pain are variable and may be exaggerated in the 9 to 11 age group and minimized in the 6 to 8 year olds (see Ped Neur Briefs April 